\title{
Avaliação comparativa em curto prazo dos parâmetros fisiológicos e variáveis cardiopulmonares em recém-nascidos internados na Unidade de Terapia Intensiva Neonatal antes e após intervenção fisioterapêutica
}

\author{
Short-term comparative assessment of physiological parameters and cardiopulmonar variables in \\ newborns admitted to the Neonatal Intensive Care Unit before and after physical therapy \\ intervention \\ Evalución comparativa a corto plazo de parámetros fisiológicos y variables cardiopulmonares em \\ recién nacidos ingresados en la Unidad de Cuidados Intensivos Neonatales antes y después de la \\ intervención de fisioterapia
}

Recebido: 21/05/2021 | Revisado: 01/06/2021 | Aceito: 04/06/2021 | Publicado: 18/06/2021

\author{
Fernanda Nolasco Nunes \\ ORCID: https://orcid.org/0000-0002-6539-6179 \\ Universidade Federal do Pampa, Brasil \\ E-mail: fernandaanolasco@gmail.com \\ Juliana Möbs Canova \\ ORCID: https://orcid.org/0000-0001-7685-7762 \\ Universidade Federal do Pampa, Brasil \\ E-mail: juuh.mcanova@gmail.com \\ Nelson Francisco Serrão Junior \\ ORCID: https://orcid.org/0000-0002-0280-0752 \\ Universidade Federal do Pampa, Brasil \\ E-mail: nelsonserrao@unipampa.edu.br \\ Jéssica Delamuta Vitti \\ ORCID: https://orcid.org/0000-0003-1987-3891 \\ Fisioterapia Campos, Brasil \\ E-mail: jehvitti@hotmail.com
}

\begin{abstract}
Resumo
Objetivo: realizar avaliação comparativa em curto prazo dos parâmetros fisiológicos e variáveis cardiopulmonares em recém-nascidos (RNs) internados em Unidade de Terapia Intensiva Neonatal (UTN) que possuem acompanhamento fisioterapêutico. Metodologia: trata-se de um estudo descritivo, comparativo, prospectivo e de análise quantitativa realizado com menores de 40 semanas de idade gestacional, internados em UTN, em abril de 2021. Os dados foram coletados a partir de prontuário eletrônico dos pacientes, entrevista com os responsáveis pelos RNs e observação dos parâmetros fisiológicos e demais variáveis cardiopulmonares, 5 minutos antes e 5 minutos após uma única intervenção fisioterapêutica. Resultados: a maioria dos recém-nascidos voluntários eram do sexo masculino $(66,67 \%)$, que nasceram via cesariana $(88,89 \%)$, de mães adultas jovens. Apenas 1 dos RNs estava em uso de suporte ventilatório não invasivo, os demais estavam em ventilação espontânea. A idade gestacional média foi de 256 dias, a média da altura foi de $44,92 \mathrm{~cm}$ e do peso ao nascer foi de $2,474 \mathrm{~g}$. A prematuridade foi o transtorno mais observado entre os participantes $(66,67 \%)$ seguidoda taquipneia transitória do recém-nascido $(44,44 \%)$. Os parâmetros fisiológicos apresentaram variações quando comparados os momentos pré e pós intervenção fisioterapêutica, a frequência cardíaca não apresentou diferença significativa $(p=0,15)$, a frequência respiratória obteve uma diferença muito significante $(p=0,008)$ e saturação periférica de oxigênio apresentou extrema significância $(\mathrm{p}=0,0008)$. Conclusão: a fisioterapia na UTIN pode colaborar nos parâmetros dos neonatos como a redução da frequência respiratória e aumento da saturação periférica de oxigênio enquanto a frequência cardíaca não sofreu alteração significativa.
\end{abstract}

Palavras-chave: Unidades de Terapia intensiva neonatal; Recém-nascidos; Fisioterapia.

\begin{abstract}
Objective: to carry out comparative evaluation in the short term of physiological parameters and cardiopulmonary variables in newborns admitted to the Neonatal Intensive Care Unit (NICU) who have physical therapy follow-up. Methodology: this is a descriptive, comparative, prospective of quantitative analysis study carried out with 9 newborns under 40 weeks of gestational age, admitted to the NICU, in April 2021. Data were collected from patients' electronic
\end{abstract}


medical records, interviews with those responsible by newborns and observation of physiological parameters and other cardiopulmonary variables, 5 minutes before and 5 minutes after a single physical therapy intervention. Results: the majority of newborn volunteers were male $(66.67 \%)$, who were born via cesarean section $(88.89 \%)$, from young adult mothers. Only 1 of the newborns was using non-invasive ventilatory support, the rest were on spontaneous ventilation. The average gestational age was 256 days, the average height was $44.92 \mathrm{~cm}$ and the birth weight was $2.474 \mathrm{~g}$. Prematurity was the most observed disorder among the participants $(66.67 \%)$, followed by the transient tachypnea of the newborn (44.44\%). The physiological parameters showed variations when comparing the moments before and after physical therapy intervention, the heart rate did not show any significant difference $(\mathrm{p}=0.15)$, the respiratory rate obtained a very significant difference $(\mathrm{p}=0.008)$ and peripheral oxygen saturation showed extreme significance $(\mathrm{p}=$ 0.0008). Conclusion: physiotherapy in the NICU can contribute to the parameters of neonates such as a reduction in respiratory rate and an increase in peripheral oxygen saturation while heart rate has not undergone any significant change.

Keywords: Intensive care units; Newborn; Physical therapy.

\section{Resumen}

Objetivo: realizar una evaluación comparativa a corto plazo de los parámetros fisiológicos y variables cardiopulmonares en los recién nacidos $(\mathrm{RN})$ ingresados en la Unidad de Cuidados Intensivos Neonatales (UTN) que tienen seguimiento de fisioterapia. Metodología: se trata de un estudio descriptivo, comparativo, prospectivo y de análisis cuantitativo realizado con 9 recién nacidos menores de 40 semanas de edad gestacional, ingresados en la UTN, en abril de 2021. Se recolectaron datos de la historia clínica electrónica de los pacientes, entrevistas a los responsables por recién nacidos. y observación de parámetros fisiológicos y otras variables cardiopulmonares, 5 minutos antes y 5 minutos después de una única intervención de fisioterapia. Resultados: la mayoría de los recién nacidos voluntarios fueron varones $(66,67 \%)$, que nacieron por cesárea $(88,89 \%)$, de madres adultas jóvenes. Solo 1 de los recién nacidos estaba usando soporte ventilatorio no invasivo, el resto estaba en ventilación espontánea. La edad gestacional promedio fue de 256 días, la altura promedio fue de 44,92 cm y el peso al nacer fue de $2,474 \mathrm{~g}$. La prematuridad fue el trastorno más observado entre los participantes $(66,67 \%)$ seguido de la taquipnea transitoria del recién nacido $(44,44 \%)$. Los parámetros fisiológicos mostraron variaciones al comparar los momentos antes y después de la intervención de fisioterapia, la frecuencia cardíaca no mostró diferencia significativa $(\mathrm{p}=0.15)$, la frecuencia respiratoria obtuvo una diferencia muy significativa $(\mathrm{p}=0.008)$ y la saturación de oxígeno periférico mostró extrema significancia $(\mathrm{p}=0,0008)$. Conclusión: la fisioterapia en la UCIN puede contribuir a los parámetros de los neonatos como una reducción de la frecuencia respiratoria y un aumento de la saturación de oxígeno periférico mientras que la frecuencia cardíaca no ha sufrido ningún cambio significativo.

Palabras clave: Unidades de cuidado intensivo neonatal; Recién-nascidos; Fisioterapia.

\section{Introdução}

A prematuridade é a principal causa de mortalidade neonatal e é considerada uma síndrome complexa, com múltiplos fatores etiológicos que estão associados a um amplo espectro de condições clínicas que podem definir a sobrevida e o padrão de crescimento e desenvolvimento de prematuros (Adams, Gomes, Strassburger, Ferreira, Colet \& Strassburger et al 2021). Calcula-se a duração média da gestação normal em 280 dias ou 40 semanas, a partir da data da última menstruação (Ministério da Saúde, 2012), já os neonatos são classificados como: atermo, quando nascidos entre 38 e 42 semanas gestacionais; prétermos quando nascidos antes de 37 semanas, ou menores que 259 dias; e pós-termos, os nascidos após 42 semanas gestacionais completas (Francisco, 2017).

De acordo com Organização Mundial da Saúde (2015), cerca de 15 milhões de bebês no mundo nascem prematuros todos os anos, sendo as complicações do parto prematuro as maiores causas de óbitos em crianças menores de cinco anos. Os fatores relacionados à prematuridade podem estar associados com as condições maternas (morbidades durante a gestação ou anteriores a ela), idade materna, alteração no aparelho genital feminino, infecções da mãe e do bebê ou até mesmo de causa desconhecida (Souza, Maia, Zêgo, Jaeger \& Maciel, 2019). Os bebês prematuros também possuem risco de desenvolver problemas graves ou complicações decorrentes de fatores neonatais, como a idade gestacional (IG), crescimento fetal e peso ao nascimento, além da influência de fatores socioeconômicos do país. O cuidado adequado de bebês com baixo peso ao nascer, como alimentação, manutenção da temperatura, cuidados higiênicos com o cordão umbilical e a pele, além de tratamento precoce de infecções e complicações, incluindo a síndrome do desconforto respiratório, pode reduzir substancialmente mortalidade dessa faixa populacional (Organização Mundial de Saúde, 2018). 
Após o nascimento, o ambiente extrauterino condiciona o neonato a uma série de adaptações à vida, que incluem a maturação morfofisiológica de diversos sistemas, principalmente do respiratório, que necessita do desenvolvimento da bioquímica do parênquima pulmonar e suas estruturas correlacionadas, fazendo com que os prematuros apresentem maior vulnerabilidade por não possuírem função pulmonar adequada, necessitando, muitas vezes, de internação em Unidade de Terapia Intensiva Neonatal (UTIN). Os distúrbios respiratórios são fatores principais associados à mortalidade (Rachuri, Oleti, Murki, Subramanian \& Nethagani, 2017). Dentre as doenças respiratórias que mais acometem os recém-nascidos pré-termo (RNPT), temos a Síndrome do Desconforto Respiratório (SDR), sendo, também, uma das principais causas de óbito, em RN com insuficiência respiratória e em prematuros de muito baixo peso. Além disso, apresenta elevada morbidade associada, tendo como uma de suas principais complicações a displasia broncopulmonar, que compromete ainda mais o prognóstico de prematuros, em curto e longo prazo (Macêdo, Leite, Cunha, Farias \& Souza, 2018). Os bebês internados podem desenvolver ou potencializar uma série de agravos ao desenvolvimento neuro-sensório-motor, sendo imprescindível a assistência multiprofissional em tempo integral (Antunes, Silva, Bocardo, Daher, Faggioto \& Rugolo et al 2006; Maia, 2016; Biazus \& Kupke, 2016).

O fisioterapeuta que atua nessa área é responsável pela avaliação fisioterapêutica e prevenção cinética funcional (de todo e qualquer sistema do corpo humano que seja necessário) assim como, por intervenções de tratamento (fisioterapia respiratória e/ou motora), atuando junto à equipe multiprofissional em alterações causadas pela síndrome de aspiração de mecônio, síndrome do desconforto respiratório, pneumonia, atelectasia, controle e aplicação de gases, ventilação pulmonar mecânica (VPM) invasiva e não invasiva (VNI), protocolos de desmame e extubação da VPM, entre outros (Antunes et al., 2006; Maia, 2016; Biazus \& Kupke, 2016; Johnston, Zanetti, Comaru, Ribeiro, Andrade \& Santos et al 2012). Somado a isso, suas técnicas promovem relaxamento muscular, posicionamento terapêutico, auto-organização sensória motora e conhecimento do próprio corpo, além de reconhecimento do ambiente extrauterino, estimulando seu desenvolvimento neuropsicomotor (Silveira, 2021).

Os avanços na qualidade da assistência e o maior acesso às ações e serviços voltados à saúde materno-infantil, juntamente com a melhoria nas condições socioeconômicas da população, têm contribuído para o acentuado declínio da mortalidade infantil no mundo (Wang, Bhutta, Coates, Coggeshall \& Dandona et al, 2016). O elevado número de óbitos verificado entre o parto e o primeiro mês de vida no Brasil, ainda representa um enorme desafio, tanto para os serviços de saúde quanto para a sociedade (Santana, Novais \& Zucchi, 2016). Existem estudos como o de Maia (2016), que abrange a atuação da fisioterapia no recém-nascido, em especial aos que necessitam de cuidados intensivos, esse profissional é capaz de proporcionar um aumento na sobrevida e reduzir sequelas sistêmicas. Porém, existem poucos exploram variáveis hemodinâmicas durante a sua atuação, por isso, o objetivo desse trabalho foi realizar uma avaliação comparativa em curto prazo dos parâmetros fisiológicos e variáveis cardiopulmonares (frequência cardíaca - FC -, frequência respiratória - FR - e saturação de oxigênio - $\mathrm{SpO}_{2}$ ) em recém-nascidos (RNs) internados na Unidade de Terapia Intensiva Neonatal antes e após intervenção fisioterapêutica.

\section{Metodologia}

O respectivo estudo foi de caráter descritivo, comparativo, prospectivo e de análise quantitativa com base em informações obtidas nas coletadas de dados. O estudo foi desenvolvido na Universidade Federal do Pampa (UNIPAMPA) e realizado no setor de Terapia Intensiva Neonatal do Hospital Santa Casa de Caridade de Uruguaiana- RS, em abril de 2021.

A coleta de dados foi iniciada após aprovação do Comitê de Ética em Pesquisa (CEP) da Universidade Federal do Pampa (UNIPAMPA) (Parecer N. 4.358.530/2019) e assinatura do Termo de Consentimento Livre e Esclarecido (TCLE) pelos 
responsáveis dos RNs participantes da pesquisa, conforme a resolução no 466/12 do Conselho Nacional de Saúde (Conselho Nacional de Saúde, 2012).

Os critérios de inclusão englobavam recém-nascidos que estavam estáveis hemodinamicamente, independentemente do suporte ventilatório (VPM, VNI) ou ventilação espontânea; e os critérios de exclusão foram RNs com extremo baixo peso $(<1000 \mathrm{~g})$ e instabilidade hemodinâmica grave.

Participaram deste estudo 9 RNs menores de 40 semanas de idade gestacional que estavam internados na Unidade de Terapia Intensiva Neonatal do Hospital Santa Casa de Caridade de Uruguaiana - RS. Os dados foram coletados a partir de anamnese, prontuário eletrônico do paciente e por meio de entrevista com os responsáveis pelos RNs, além da observação dos parâmetros fisiológicos e demais variáveis cardiopulmonares. As variáveis fisiológicas mensuradas foram: $\mathrm{FC}$ e $\mathrm{SpO}_{2}$ a partir do monitor multiparamétrico localizado beira leito e FR através da contagem das incursões respiratórias (movimentos toracoabdominais) realizadas durante 1 minuto. Os registros foram coletados 5 minutos antes e 5 minutos após uma única intervenção fisioterapêutica realizada pela manhã, por uma única fisioterapeuta do setor, evitando alterações na rotina dos cuidados dos neonatos. As intervenções fisioterapêuticas utilizadas na pesquisa foram a vibrocompressão, troca de posicionamentos, o método de reequilíbrio toracoabdominal (RTA), estimulação tátil cinestésica e a cinesioterapia.

A vibrocompressão é uma manobra de higiene brônquica realizada aplicando-se movimentos oscilatórios manuais no tórax do paciente. As manobras de higiene brônquica são técnicas que visam auxiliar na mobilização e remoção das secreções retidas, prevenir ou reduzir as obstruções por secreção e suas consequências (Draghi, Manzano \& Ambrozin, 2018). O método reequilíbrio toracoabdominal é uma técnica que tem por objetivo incentivar a ventilação pulmonar e promover a remoção de secreções pulmonares e de vias aéreas superiores, através da reorganização do sinergismo muscular respiratório, que se perde nas doenças pulmonares. É baseado em posicionamentos, mobilizações das articulações costovertebrais e costocondrais, alongamentos musculares, apoios manuais para aumentar a pressão intra-abdominal e manobras miofasciais, conduzidos para minimizar o uso da musculatura acessória da inspiração e da expiração, reeducando o trabalho respiratório que se encontra sobrecarregado nas patologias neonatais (Rousenq, Scalco, Rosa, Honório \& Schivinski, 2013). Já o posicionamento é o ato de mudar a posição do bebê a fim de melhor acomodá-lo ou simplesmente mudá-lo de decúbito para prevenir escaras (Cardoso, Lima, Maximino \& Specian, 2018). Podendo ocorrer a mudança de posição como em prona, em ninho, com a utilização de redes, decúbito dorsal e outros. No estudo de Costa, Beleza, Souza \& Ribeiro (2016) a rede favoreceu a postura flexora, promovendo melhor organização do bebê e o posicionamento em decúbito dorsal em uma rede foi associado a uma maior maturidade neuromuscular. A estimulação cinestésica, tátil e vestibular são alguns dos métodos para se promover o desenvolvimento, além de minimizar a dor, estabilizar o padrão motor e o tônus, uma resposta comportamental mais adequada e até mesmo incrementar o ganho de peso (Oliveira, Mendonça \& Freitas, 2015). Esta conduta favorece o crescimento de células cerebrais, que favorece o melhor desenvolvimento da criança e sua capacidade de adaptação (Aliabadi \& Askary, 2013).

\section{Resultados}

Foram avaliados o total de 9 RNs internados na UTIN, filhos de mães adultas jovens, com a idade média de 26,7 $\pm 6,6$ anos (idade mínima 14 e máxima 35 anos), nascidos predominantemente via cesariana (88,89\%). Quanto às intercorrências durante a gestação $(66,67 \%)$ das mães tiveram, sendo a infecção do trato urinário (ITU) a patologia mais incidente, seguida de doença hipertensiva específica da gravidez (DHEG), diabetes gestacional, placenta prévia, bolsa rota e restrição do crescimento intrauterino e destas mães $(33,33 \%)$ não apresentaram intercorrências durante a gravidez. A descrição completa dos dados se encontra na Tabela 1. 
Tabela 1. Características descritas do perfil materno de acordo com a idade, via de parto e intercorrências durante a gravidez.

\begin{tabular}{lll}
\hline \multicolumn{1}{c}{ Característica } & $\mathbf{N}$ & $(\boldsymbol{\%})$ \\
\hline \hline Idade materna & & \\
\hline 12 a 17 & 1 & $11,11 \%$ \\
18 a 24 & 1 & $11,11 \%$ \\
25 a 35 & 7 & $77,78 \%$ \\
Via de parto & & \\
\hline Cesárea & 8 & $88,89 \%$ \\
Normal & 1 & $11,11 \%$ \\
Intercorrências na gravidez & & $66,67 \%$ \\
\hline Sim & 6 & $33,33 \%$ \\
Não & 3 & \\
\hline
\end{tabular}

Legenda: N= número de mães; \%= porcentagem de acordo com o número de mães. Fonte: Nunes e Canova (2021).

Os voluntários eram predominantemente do sexo masculino (66,67\%), com menos de 40 semanas de idade gestacional (em média 33,7 semanas) (236 dias \pm 29,41), variando de 27 semanas e 5 dias a 38 semanas e 5 dias; e idade gestacional corrigida, média de 36,6 semanas (258 dias $\pm 21,28)$. Os dados de comprimento dos bebês foram em média de $44,92 \mathrm{~cm}(44,92 \pm 2,13)$ e o peso ao nascer médio de $2,474 \mathrm{~g}(2,474 \pm 0,73)$, variando de $1628 \mathrm{~g}$ a $3660 \mathrm{~g}$. Oito dos participantes estavam sem suporte ventilatório (ar ambiente) e somente 1 estava com suporte ventilatório não invasivo.

Os neonatos apresentaram diferentes hipóteses diagnósticas acerca de suas patologias, tais como: prematuridade $(66,67 \%)$, taquipneia transitória do recém-nascido (TTRN) $(44,44 \%)$, icterícia $(33,33 \%)$, sepse $(33,33 \%)$, síndrome do desconforto respiratório (SDR) $(22,22 \%)$ e displasia broncopulmonar (DBP) $(11,11 \%)$. Essas informações foram descritas nas Tabelas 2,3 e 4 .

Tabela 2. Fatores dos recém-nascidos de acordo com a média da idade gestacional, altura e peso ao nascer.

\begin{tabular}{|c|c|}
\hline Variáveis & $\mathbf{M} \pm \mathbf{D P}$ \\
\hline IG (dias) & $236 \pm 29,41$ \\
\hline IGC (dias) & $258 \pm 21,28$ \\
\hline Altura $(\mathrm{cm})$ & $44,92 \pm 2,13$ \\
\hline Peso ao nascer (g) & $2,474 \pm 0,73$ \\
\hline
\end{tabular}


Tabela 3. Demais características do neonato internado na UTIN de acordo com o sexo e a classificação do peso ao nascer.

\begin{tabular}{ll}
\hline \multicolumn{1}{c}{ Variáveis } & N $(\boldsymbol{\%})$ \\
\hline \hline Sexo & $6(66,67 \%)$ \\
\hline Masculino & $3(33,33 \%)$ \\
Feminino & \\
\hline Classificação peso ao nascer, n (\%) & $6(66,67 \%)$ \\
\hline 1500 a 2500g & $3(33,33 \%)$ \\
Superior a 2500g & \\
\hline
\end{tabular}

Legenda: $\mathrm{N}=$ número de recém-nascidos; \%= porcentagem de acordo com o número de recém-nascidos; $\mathrm{g}=$ gramas. Fonte: Nunes e Canova (2021).

Tabela 4. Descrição dos diagnósticos coletados de acordo com cada prontuário dos recém-nascidos internados na UTIN, sendo a provável indicação para intervenção fisioterapêutica além da prematuridade.
Variável
(N)
$(\%)$

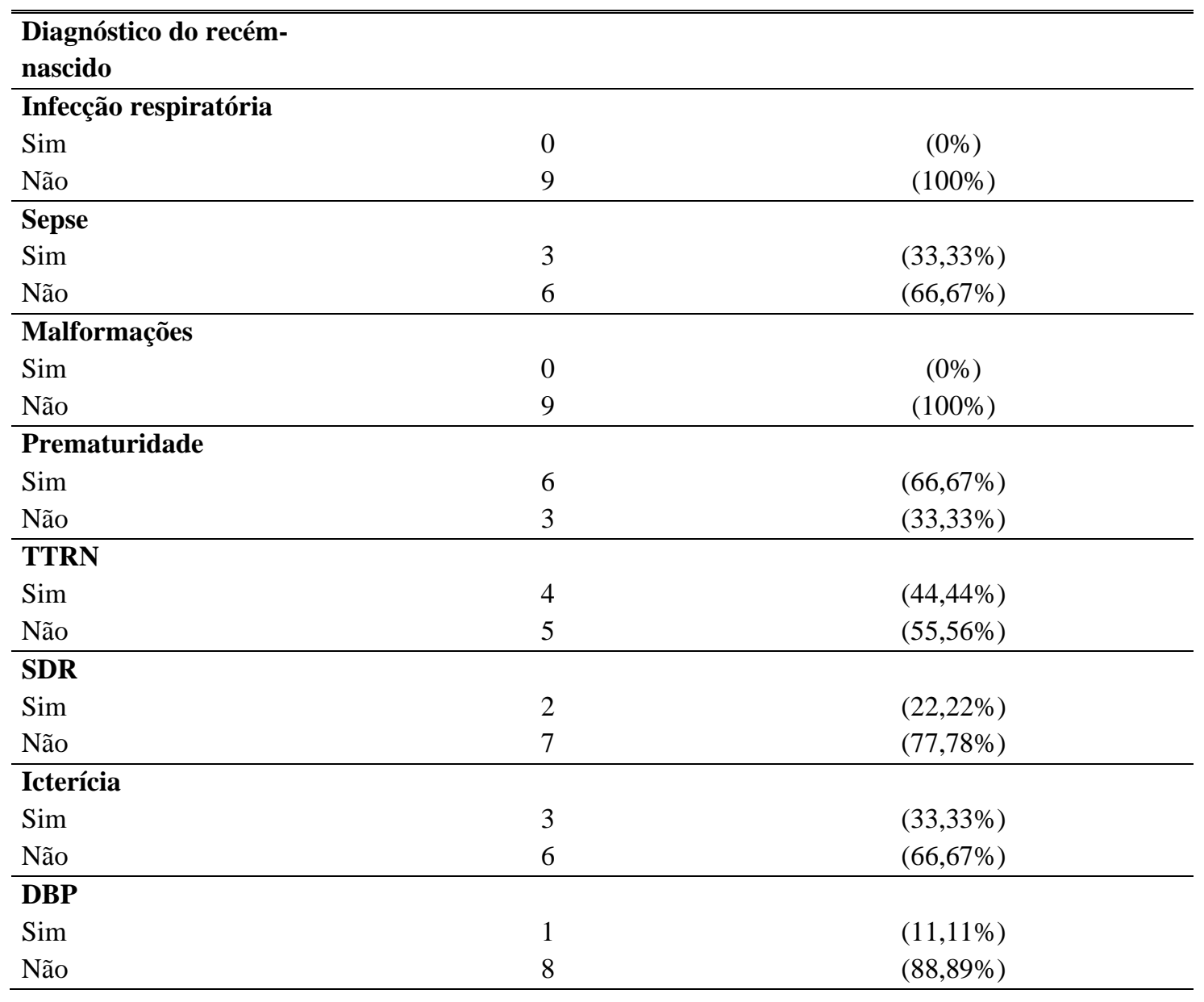

Legenda: TTRN=Taquipneia transitória do recém-nascido; SDR= Síndrome do desconforto respiratório; DBP= displasia broncopulmonar; $\mathrm{N}=$ número de recém-nascidos, \%= porcentagem de acordo com o número de recémnascidos. Fonte: Nunes e Canova (2021). 
A intervenção fisioterapêutica demonstrou diferença em curto prazo sobre os parâmetros de frequência cardíaca, frequência respiratória e saturação periférica de oxigênio em todos os recém-nascidos avaliados. Foi definido momento 1 (M1) como a primeira coleta de dados realizada 5 minutos antes do atendimento fisioterapêutico e momento 2 (M2), a coleta de dados após 5 minutos o atendimento, para comparação desta prática (antes e depois). A variável frequência cardíaca diminuiu em todos os participantes do estudo em relação aos momentos 1 e 2 (M1 e M2), porém sem diferença estatisticamente significante $(\mathrm{p}=0,15)$. Na frequência respiratória houve uma diminuição em todos os recém-nascidos entre o M1 e M2, obtendo diferença estatisticamente significante $(p=0,008)$ e a saturação periférica de oxigênio também apresentou diferença significante $(\mathrm{p}=0,0008)$ entre os momentos 1 e 2 (M1 e M2). Os resultados foram detalhados na Tabela 5.

Tabela 5. Demonstra a diferença dos parâmetros $\mathrm{FC}$, FR e $\mathrm{SpO}_{2}$ em dois momentos de acordo com a intervenção fisioterapêutica da UTIN.

\begin{tabular}{cccc}
\hline Variável & M1 & M2 & Valor p \\
\hline \hline Parâmetros fisiológicos & M $\pm \mathbf{D P}$ & $\mathbf{M} \pm \mathbf{D P}$ & $\mathbf{p}^{*}$ \\
\hline $\mathrm{FC}$ & $149 \pm 21,94$ & $143,5 \pm 18,51$ & 0,15 \\
\hline $\mathrm{FR}$ & $55,5 \pm 8,18$ & $53,4 \pm 8,01$ & 0,008 \\
\hline $\mathrm{SpO}_{2}$ & $95,7 \pm 2,4$ & $99 \pm 0,92$ & 0,0008 \\
\hline
\end{tabular}

Legenda: $\mathrm{FC}=$ Frequência cardíaca; $\mathrm{FR}=$ frequência respiratória; $\mathrm{SpO}_{2}=$ Saturação periférica de oxigênio; $\mathrm{M} 1=$ momento anterior a intervenção fisioterapêutica (5 minutos antes); $\mathrm{M} 2=$ momento após a intervenção fisioterapêutica (5 minutos após); $\mathrm{M}=$ média da amostra; $\mathrm{DP}=$ desvio padrão; $\mathrm{p}<0,05=$ valor de significância. Fonte: Nunes e Canova (2021).

\section{Discussão}

O respectivo estudo demonstrou prevalência da prematuridade, taquipneia transitória do RN, baixo peso ao nascer, gênero masculino e via de parto dominante cesariana, em RNs internados em UTIN. As intercorrências maternas estavam relacionadas à infecção do trato urinário, a doença hipertensiva da gravidez, placenta prévia, bolsa rota e restrição de crescimento. A variação da frequência cardíaca entre o momento pré fisioterapia (M1) e o momento após a fisioterapia (M2) teve uma diminuição dos seus valores, porém não foi foram significantes. A frequência respiratória resultou em uma diminuição quando comparado entre os momentos pré (M1) e pós fisioterapia (M2), tendo uma variação significativa e o parâmetro da saturação periférica entre esses momentos da intervenção fisioterapêutica houve um aumento entre os registros pré (M1) e pós fisioterapia (M2), sugerindo relevâncias fisiológicas positivas agudas nos RN prematuros que receberam atendimento na UTIN. Deste modo, os fisioterapeutas são capazes de intervir na instabilidade hemodinâmica, buscando observar os efeitos da atividade terapêutica sobre os parâmetros fisiológicos, ventilatórios, tempo de internação e suas complicações, otimizando as funções do organismo e promovendo o desenvolvimento desse prematuro (Duarte, 2011).

A prematuridade é caracterizada uma condição muito comum e no presente estudo apresentou-se com 66,67\%. O trabalho de Brandi, Rocha, Silva, Bretas, Rodrigues e Araujo et al (2020) apontam que a infecção do trato urinário constituiu um fator para risco de prematuridade, seguido da doença hipertensiva da gravidez, diabetes melittus gestacional e a restrição do crescimento intrauterino, fatores também observados no presente estudo. Já Rodrigue e Belham (2017) concluíram que a interrupção da gestação foi predominantemente motivada por doença hipertensiva da gestação e suas complicações, em $45 \%$ dos casos, seguido de diabetes melittus gestacional em $29 \%$ das gestantes, ao avaliarem o perfil de recém-nascidos na Unidade de Terapia Intensiva Neonatal, divergindo dos achados do presente estudo. No presente estudo o peso ao nascer médio dos 
recém-nascidos participantes foi de $2474(2,474 \pm 0,73)$, variando de $1628 \mathrm{~g}$ a $3660 \mathrm{~g}$. Os neonatos com peso ao nascer $<2500 \mathrm{~g}$ apresentaram quatro vezes mais chances de nascimento prematuro (Passini, Cecatti, Lajos, Tedesco, Nomura \& Haddad et al 2014).

Silveira, Tavella, Fernandez, Ribeiro, Garcia e Júnior et al (2020), analisaram 3 Unidades de Terapias Intensiva Neonatal de diferentes hospitais localizados no estado do Rio Grande do Sul, totalizando uma amostra de 254 recém-nascidos, em que os bebês apresentavam predominantemente prematuridade, baixo peso ao nascer e doenças respiratórias, respectivamente. Granzotto, Fonseca e Lindemann (2012), avaliaram 172 recém-nascidos e observaram predomínio de taquipneia transitória do recém-nascido, síndrome do desconforto respiratório, sepse e displasia broncopulmonar, fatores também observados nesta pesquisa entre os diagnósticos encontrados nos recém-nascidos voluntários, sendo a taquipneia transitória do recém-nascido com $(44,44 \%)$, síndrome do desconforto respiratório $(22,22 \%)$, sepse $(33,33 \%)$ e displasia broncopulmonar (11,11\%). O estudo de revisão de Bittencourt (2017) analisou que a utilização da vibrocompressão e reequilíbrio toracoabdominal (RTA), durante o atendimento fisioterapêutico nos RNs internados na UTIN, mostraram estabilidade hemodinâmica, sem mudanças significativas nos parâmetros fisiológicos, não indicando riscos aos neonatos. Assim como, a pesquisa de Giannantonio (2010) observou o aumento nos valores de saturação periférica de oxigênio, sem evidencias de efeitos significativos na FC, corroborando ao presente estudo, onde a saturação demonstrou diferença estatisticamente significante ( $\mathrm{p}=0,0008)$ e a $\mathrm{FC}$ não apresentou diferença significativa $(\mathrm{p}=0,15)$.

Roussenq et al (2013) verificaram a $\mathrm{FR}, \mathrm{FC}$ e $\mathrm{SpO}_{2}$ em $24 \mathrm{RNs}$, antes e após a fisioterapia, observando uma diminuição significativa da FR nos RN submetidos a intervenção fisioterapêutica. Já no estudo de Martins, Silva, Honório, Paulin e Schivinski (2013b), os autores avaliaram os parâmetros cardiorrespiratórios ( $\mathrm{SpO}^{2}$, FC e FR) de 14 RNs que receberam apenas uma intervenção fisioterapêutica, em que se observou uma queda estatisticamente significativa da FC, contrastando ao achado desta pesquisa, pois a diferença foi de $(\mathrm{p}=0,15)$. As variações de $\mathrm{FC}$ e FR podem ocorrer devido às manipulações e procedimentos que os recém-nascidos recebem durante a internação, sendo o excesso de estimulação prejudicial, pois promove aumento do gasto energético (Padilha \& Bombarda, 2020).

Na pesquisa realizada por Silva, Costa, Figueiredo, Menezes, Gandra e Rodrigues et al (2020) abordaram acerca do método canguru na UTIN, onde foram observados benefícios como a estabilização cardiorrespiratória, regulação da frequência respiratória e melhora da oxigenação sendo considerados parâmetros significativos corroborando com este trabalho quando realizadas técnicas de fisioterapia como a troca de posicionamentos e RTA. Olmedo, Gabas, Merey, Souza, Muller \& Santos et al (2012) avaliaram e compararam as respostas fisiológicas (frequência cardíaca, frequência respiratória, saturação arterial de oxigênio e a temperatura corporal) também utilizando o método canguru associado a posição prona em recém-nascidos prétermo (RNPT), no qual foram observados diminuição da frequência respiratória após duas intervenções em momentos distintos e aumento da $\mathrm{SatO}_{2}$ no $3^{\circ}$ dia após a fisioterapia, corroborando aos achados deste estudo, apesar da diferente metodologia apresentada, pois neste foi realizado após uma única intervenção e demonstrando resultados significativos em curto prazo.

Martins, Silva, Honório, Paulin e Schivinski (2013) avaliaram 60 RNs divididos em três grupos: G1- grupo controle e os grupos que receberam fisioterapia respiratória, sendo G2 a fisioterapia convencional e G3 o método reequilíbrio toracoabdominal. O estudo observou os parâmetros cardiovasculares (saturação periférica de oxigênio, frequência cardíaca, frequência respiratória), antes da intervenção, imediatamente após e 15 minutos após a intervenção fisioterapêutica, como resultado não obtiveram alterações significativas na saturação periférica, na frequência cardíaca e na frequência respiratória avaliada, refutando os achados com o presente estudo, pois este não possuiu grupo controle, além da medida de tempo avaliada ter sido diferente o que pode ter relacionado com o retorno dos valores basais.

No estudo de Lanza, Kim, Silva, Vasconcelos e Tsopanoglou (2010) foram avaliados os parâmetros fisiológicos 5 minutos antes da fisioterapia, 5 minutos após o início da fisioterapia e 30 minutos após o término a intervenção, em 16 recém- 
nascidos prematuros na UTI, onde não foram demonstradas mudanças significativas das variáveis fisiológicas observadas (FC, FR e $\mathrm{SpO}_{2}$ ), divergindo do presente estudo, devido a diferente metodologia realizada quanto ao tempo de coleta. Da mesma forma, Tavares, Treichel, Ling, Scopel e Lukrafka (2019) avaliaram as alterações fisiológicas (frequência cardíaca, frequência respiratória, saturação periférica de oxigênio e temperatura) em 30 neonatos com diagnósticos de síndrome do desconforto respiratório e sem suporte ventilatório, o protocolo foi realizado em três momentos da intervenção fisioterapêutica, antes do atendimento, imediatamente após a intervenção e 15 minutos após o final do atendimento. No estudo observou-se o aumento significativo na frequência cardíaca imediatamente após o protocolo, quando comparados os três momentos. Porém, com retorno aos valores basais 15 minutos após a fisioterapia, as outras variáveis fisiológicas ( $\mathrm{FR}, \mathrm{SpO}_{2}$ e temperatura) não apresentaram alterações significativas.

Abreu, Oliveira, Leone, Siqueira, Herreiro e Wajnsztejn et al (2011) também analisaram as mesmas variáveis hemodinâmicas em 44 RNs com SDR (sem suporte ventilatório) no momento pré fisioterapia (2 minutos) e depois (5 minutos) do atendimento fisioterapêutico onde apresentou resultados significativos. A relação da intervenção respiratória e mobilização mostraram-se positiva na diminuição da frequência cardíaca, da pressão arterial sistólica e da pressão arterial média, no entanto sem resultados significativos na frequência respiratória, saturação periférica de oxigênio e temperatura corporal.

O presente estudo apresentou algumas limitações que incluem o número da amostra reduzido, ausência de grupo controle e o tempo de coleta, limitado pela pandemia da COVID-19 devido ao alto número de casos na cidade de Uruguaiana no Rio Grande do Sul e por ter um acesso mais restrito à entrada ao hospital pelo risco de contaminação, o que foram fatores restritivos para considerações e conclusões mais específicas dos dados obtidos na pesquisa. Porém, sabe-se que os fisioterapeutas possuem um papel importante no que diz respeito a controlar as instabilidades fisiológicas e o tratamento deste profissional promove a estabilidade das variáveis hemodinâmicas do RN (Abreu et al. 2011).

$\mathrm{O}$ atendimento fisioterapêutico promove resultados que podem ser observados na redução ou alívio da dor, correção de posicionamento, bem como uma sensação de bem-estar sob condições críticas de internação. As intervenções e os tipos de condutas do fisioterapeuta podem variar de local para local e da equipe da UTIN e podem auxiliar na diminuição da mortalidade e morbidade dos RN internados. A fisioterapia desempenha um papel importante dentro das UTINs, promovendo a qualidade de vida dos RN e também de seus familiares (Theis, Gerzson \& Almeida, 2016).

\section{Conclusão}

Este estudo evidenciou que a fisioterapia alterou significativamente os parâmetros de frequência respiratória ocasionando a diminuição dessa variável e ocasionou o aumento da saturação periférica de oxigênio de forma significativa nos RNs internados na UTIN, quando comparados os momentos 1 e 2, não apresentando resultados significativos sobre a variável frequência cardíaca.

Ademais, recomendam-se novos estudos para evidenciar a importância da atuação do fisioterapeuta dentro das UTINs que possam comprovar mudanças significativas dos parâmetros fisiológicos dos neonatos, quando aplicados em diferentes mensurações de tempo, a curto e longo prazo, além de outras técnicas fisioterapêuticas realizadas.

\section{Referências}

Abreu, L. C., Valenti, V. E., Oliveira A. G., Leone, C., Siqueira, A. A, Herreiro, D., Wajnsztejn, R., Manhabusque, K. V., Júnior, H. M., Mello, M. C. B., Fernandes L. L. \& Saldiva, P. H. (2011). Chest associated to motor physiotherapy improves cardiovascular variables in newborns with respiratory distress syndrome. Int Arch Med. 37(4). 1755-1776. 10.1186/1755-7682-4-37.

Adams, F. C., Gomes, J. S., Strassburger, M. J., Ferreira P. F., Colet, C. F. \& Strassbuger S. Z. (2021). Fatores associados à idade gestacional de prematuros internados em unidade de terapia intensiva neonatal. Revista Eletrônica de Ciência, Tecnologia e Inovação em Saúde. 13(1), 158-163. 10.9789/21755361.rpcfo.v13.8069. 
Aliabadi F. \& Askary R. (2013). Effects of tactile-kinesthetic stimulation on low birth weight neonates. Iran J Pediatr. 23(3), 289-294. https://www.ncbi.nlm.nih.gov/pmc/articles/PMC3684473/.

Antunes, L. C. O., Silva, E. G., Bocardo, P., Daher, D. R., Faggioto, R., \& Rugolo, L. M. S. S. (2006). Efeitos da fisioterapia respiratória convencional versus aumento do fluxo expiratório na saturação de $\mathrm{O} 2$, frequêencia cardíaca e frequiência respiratória, em prematuros no período pós-extubação. Brazilian Journal of Physical Therapy, 10(1), 97-103. 10.1590/S1413-35552006000100013.

Biazus, G. F., \& Kupke, C. C. (2016). Clinical profile of newborns undergoing physical therapy in a neonatal intensive care unit. Fisioterapia em Movimento, 29(3), 553-560. 10.1590/1980-5918.029.003.AO13.

Bittencourt, D. (2017). Técnicas de fisioterapia respiratória na Unidade de Terapia Intensiva Neonatal. Revista saúde integrada. 10(19), 2-15. http://local.cnecsan.edu.br/revista/index.php/saude/index.

Brandi, L.D.A., Rocha, L.R., Silva, L.S., Bretas, L.G., Rodrigues, M.A. \& Araújo, S.T.H. (2020) Fatores de risco materno-fetais para o nascimento pré-termo em hospital de referência de Minas Gerais. Revista Médica de Minas Gerais. 30(sup14), 41-47. http://rmmg.org/exportar-pdf/2701/v30s04a07.pdf.

Brasil. Ministério da Saúde. Atenção ao pré-natal de baixo risco. Cadernos de Atenção Básica. http://dab.saude.gov.br/portaldab/biblioteca.php?conteudo=publicacoes/cab32.

Cardoso, A. S., Lima, A. M., Maximino, V. S. \& Specian, C. M. (2010). Estudo exploratório de dor em recém-nascidos prétermos em uma unidade de tratamento intensivo neonatal. Cadernos de Terapia Ocupacional da UFSCar. 18(2), 105-114. http://www.cadernosdeto.ufscar.br/index.php/cadernos/article/view/349/278.

Costa, K. S. F., Beleza, L. O., Souza, L. M., \& Ribeiro, L. M. (2016). Rede de descanso e ninho: comparação entre efeitos fisiológicos e comportamentais em prematuros. Revista Gaúcha de Enfermagem, 37(spe), e62554. 10.1590/1983-1447.2016.esp.62554

Draghi, T. T., Manzano, R. M. \& Ambrozin, A. R. P. (2018). Efeitos do instrumento Pulsar® e da vibrocompressão em crianças no ambiente hospitalar. ConScientiae Saúde. 17(1), 86-92. 10.5585/conssaude.v17n1.7955.

Francisco, P. (2017). Abordagem fisioterapêutica em prematuro de alto risco na UTI neonatal. Revista Fisioterapia Brasil. 9(6), 422-426. 10.33233/fb.v9i6.1733.

Giannantonio, C. (2010). Chest physiotherapy in preterm infants with lung diseases. Italian Journal of Pediatrics, 36(1), 60-65. 10.1186 / 1824-7288-36-65.

Granzoto, J., Fonseca, S. S. \& Lindemann, F. L. (2012). Fatores relacionados com a mortalidade neonatal em uma Unidade de Terapia Intensiva neonatal na região Sul do Brasil. Revista da Associação Médica do Rio Grande do Sul. 56 (1), 57-62. https://pesquisa.bvsalud.org/portal/resource/pt/li1-647293.

Johnston, C., Zanetti, N. M., Comaru, T., Ribeiro, S. N. S., Andrade, L. B., \& Santos, S. L. L. (2012). I Recomendação brasileira de fisioterapia respiratória em unidade de terapia intensiva pediátrica e neonatal. Revista Brasileira de Terapia Intensiva, 24(2), 119-129. 10.1590/S0103-507X2012000200005.

Lanza, F. C., Kim, A. H. K., Silva, J. L., Vasconcelos, A. \& Tsopanoglou, S. P. (2010). A vibração torácica na fisioterapia respiratória de recém-nascidos causa dor? Revista Paulista de Pediatria. 28(1), 10-4. 10.1590/S0103-05822010000100003.

Macêdo, B. L. N., Leite, I. N. M. R., Cunha, T. M., Farias, C. A. C. \& Souza, V. F. (2018). Perfil epidemiológico de recém-nascidos com síndrome do desconforto respiratório e sua comparação com taxa de mortalidade. ASSOBRAFIR Ciência. 9(2), 33-43. https://www.cpcrjournal.org/article/5da73af70e8825925fba68e1.

Maia, F. (2016). A fisioterapia nas unidades de terapia intensiva neonatal. Revista da Faculdade de Ciências Médicas de Sorocaba, 18(1), 64-65. $10.5327 / Z 1984-4840201622134$.

Martins, R., Silva, M. E. M., Honório, G. J. S., Paulin, E. \& Schivinski, C. I. S. (2013a). Técnicas de fisioterapia respiratoria: efeito nos parâmetros cardiorrespiratórios e na dor do neonato estável em UTIN. Revista Brasileira de Saúde Materna e Iinfantil. 13(4), 317-327. 10.1590/S151938292013000400004

Martins, R., Silva, M. E. M., Honório, G. J. S., Paulin, E. \& Schivinski, C. I. S. (2013b). Fisioterapia respiratória no neonato estável em UTIN: comparação entre técnicas. Revista Pediatria Moderna, 49(12), 547-552b. https://pesquisa.bvsalud.org/portal/resource/pt/lil-712190.

Oliveira B. S., Mendonça K. M. P. P. \& Freitas D. A. (2015). Fisioterapia motora no recém-nascido prematuro em Unidade Intensiva Neonatal: uma revisão sistemática. ConScientiae Saúde. 14(4), 647-654. 10.5585/conssaude.v14n4.5254.

Olmedo, M. D., Gabas, G. S., Merey, L. S. F., Souza, L. S., Muller, K. T. C., Santos, M. L. M. \& Marques, C. F. (2012). Respostas fisiológicas de recémnascidos pré-termo submetidos ao método mãe-canguru e a posição prona. Revista Fisioterapia e Pesquisa. 19(2), 115-121. 10.1590/S180929502012000200005

Padilha, K. B. \& Bombarda, A.(2020). Estimulação tátil-cinestésica em recém-nascido pré-termo. Revista Ciência \& Humanização Hospital de Clínicas de Passo Fundo. 1(1), 22-37. https://rechhc.com.br/index.php/rechhc/article/view/26/25.

Passini, R., Cecatti, J. G., Lajos, G. J, Tedesco R. P., Nomura M. L., Dias, T. Z., Haddad S. M., Rehder, P. M., Pacagnella, R. C., Costa, M. L. \& Sousa, M. H. (2014). Brazilian multicentre study on preterm birth (EMIP): prevalence and factors associated with spontaneous preterm birth. Plos one 9(10), e109069. 10.1371/journal.pone.0109069.

Pereira, A. S. et al. (2018). Metodologia da pesquisa científica. Santa Maria, Rio Grande do Sul. Núcleo de Tecnologia Educacional.

Rachuri, H., Oleti, T. P., Murki, S., Subramanian, S. \& Nethagani, J. (2017). Diagnostic Performance of Point of Care Ultrasonography in Identifying the Etiology of Respiratory Distress in Neonates. Indian J Pediatr. 84(4), 267-270. 10.1007 / s12098-016-2288-7. 
Resolução $n^{\circ}$ 466/12 (2012). Dispõe sobre diretrizes e normas regulamentadoras de pesquisas envolvendo seres humanos. Diário Oficial da República Federativa do Brasil. Brasília, DF. http://bit.ly/1mTMIS3.

Rodrigue, V. B. \& Belham A. (2017) Perfil dos recém-nascidos admitidos na UTI neonatal do hospital Santo Antônio, Blumenau/SC, entre 2014-2016. Arquivo Catarinense de medicina. 46(4), 43-49. http://www.acm.org.br/acm/seer/index.php/arquivos/article/view/188.

Roussenq, K. R., Scalco, J. C., Rosa, G. J., Honório, G. J. S. \& Schivinski, C. I. S. (2013). Reequilíbrio tóraco-abdominal em recém-nascidos prematuros: efeitos em parâmetros cardiorrespiratórios, no comportamento, na dor e no desconforto respiratório. Revista Acta Fisiátrica. 20(3), 118-123. 10.5935/01047795.20130019

Santana, M. P. S., Novais, A. P. M. \& Zucchi, P. (2016). Internações hospitalares de neonatos com síndrome do desconforto respiratório e sua participação nas internações hospitalares neonatais no âmbito do Sistema Único de Saúde em 2015. Int. J. Healthc Manag. 2(1), 1-18. 10.21902/jhmreview.v2i1.321.

Silva, A. S., Costa, J. P., Figueiredo, L. S. M., Menezes, J. V., Gandra, V. D., Rodrigues, T. D. N., Silva, F. J. S. \& Domingos, L. L. P. (2020). A importância do método mãe canguru na unidade de terapia intensiva neonatal: uma revisão de literatura. Revista Brasileira de Terapia e Saúde. 10(2), 1-6. 10.7436/rbts2020.10.02.01.

Silveira, L. M. B. (2021). Benefícios da estimulação sensório motora em neonatos prematuros. Interfisio. Abril, 2021.https://interfisio.com.br/beneficios-daestimulacao-sensorio-motora-em-neonatos-prematuros/.

Silveira, T. B., Tavella, R. A., Fernandez, J. B., Ribeiro, A. P. F. A, Garcia, E. M. \& Júnior, F. M. R. S. (2020). Perfil epidemiológico de recém-nascidos internados em Unidades de Terapia Intensiva Neonatal em hospitais universitários no extremo Sul do Brasil. Revista de Ciências da Saúde 32(2), 46-54. 10.14295/vittalle.v32i2.9815

Souza, D. M. L, Maia, L. C. S., Zêgo Z. D. F., Jaeger G. P., \& Maciel, W. S. (2019). Prevalência de prematuridade e fatores associados no estado do Rio Grande Do Sul. Journal Brazilian of Health Review. 2(5), 4052-4070. 10.34119/bjhrv2n5-014.

Tavares, A. B., Treichel, L., Ling, C. C., Scopel, G. G., \& Lukrafka, J. L. (2019). Fisioterapia respiratória não altera agudamente os parâmetros fisiológicos ou os níveis de dor em prematuros com síndrome do desconforto respiratório internados em unidade de terapia intensiva. Revista Fisioterapia e Pesquisa. 26(4), 373-379. 10.1590/1809-2950/18020126042019.

Theis, R. C. S. R., Gerzson, L. R. \& Almeida, C. S. (2016). A atuação do profissional fisioterapeuta em unidades de terapia intensiva neonatal. Revista Cinergis, 17(2), 01- 09. 10.17058/cinergis.v17i2.7703.

WHO (2018). Maternal, newborn child and adolescente health. https://www.who.int/maternal_child_adolescent/newborns/prematurity/en/.

WHO (2015). Preterm birth. https://www.who.int/en/news-room/fact-sheets/detail/preterm-birth. 